\title{
ON THE INTERIOR BALLISTICS OF AN UNDERWATER PERSONAL GUN
}

\author{
Nguyen Sy Hoa ${ }^{1}$, Tran Van Tran ${ }^{2, *}$, Tran Thu $\mathrm{Ha}^{3}$ \\ ${ }^{1}$ Le Quy Don Technical University, Hanoi, Vietnam \\ ${ }^{2}$ VNU University of Science, Hanoi, Vietnam \\ ${ }^{3}$ Institute of Mechanics, VAST, Hanoi, Vietnam \\ *E-mail: trantv@vnu.edu.vn \\ Received December 02, 2015
}

\begin{abstract}
This paper deals with solving the problem of interior ballistics for a concrete personal underwater gun. The governing equations of the problem are carefully discussed because the water resistance force acting on the bullet is quite different from that of the air. These equations are used to create software for analyzing and designing an underwater gun. The calculated results are in very good agreement with the experimental data.
\end{abstract}

Keywords: Interior ballistics, underwater, gun, muzzle velocity, propellant charge.

\section{INTRODUCTION}

In practice every gun has been created for some early chosen operational aim in some specific fighting conditions. All these factors lead to the designing the geometrical form of the gun and its bullet that might make the gun more efficient in use for reaching the chosen aim. When the geometrical sizes and weight of the gun and the bullet are defined the interior ballistics should work out a propellant charge that will give the bullet the desired muzzle velocity without any damage to the weapon.

The theory of ballistics including both the interior and exterior ballistics had started long ago. Before the end of the World War II the theory was mainly developed for guns of different caliber. Next the ballistic theory has been expanded for rockets. Fundamentals of the theory of ballistics can be found in numerous books such as [1-4].

Ballistic calculations for underwater weapon are not as popular as for guns used on the ground. The main difficulty of underwater ballistic calculations relates to the high density of the environment. This paper deals with only interior ballistic problem for a underwater personal gun. Let $x$ be the position of the base of the bullet in the barrel tube (see Fig. 1), $V$ is its velocity. The governing equations of the interior ballistic problem 

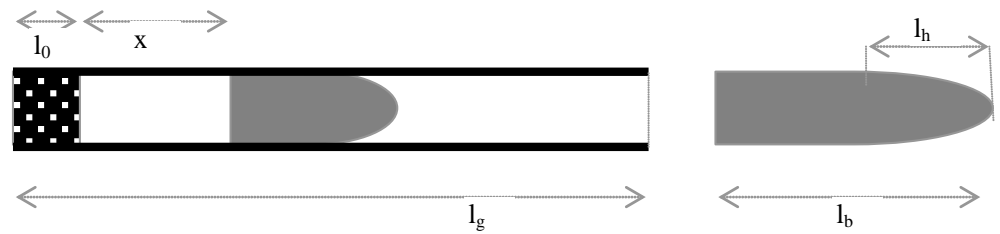

Fig. 1. Characteristics of the gun and bullet

look like that

$$
\left\{\begin{array}{l}
\dot{x}=V \\
\left(q+m_{a d}\right) \dot{V}=p_{S} S-R
\end{array}\right.
$$

where the dot denotes the derivative respect to $t, q$ is the bullet mass, $m_{a d}$ is the so-called added mass that represents the water mass expelled by the motion of the bullet from the barrel at moment $t . p_{s}$ is the pressure of the gas on the bullet base, the area of which is $S$, $R$ is the total resistance force acted on the bullet when it moves in the barrel. To solve (1) we should work out the dependence of $p_{s}$ and $R$ on $t$ or on $x$.

\section{THE PROBLEM DESCRIPTION}

The gun design is shown in Fig. 1. As mentioned above when such parameters as the barrel size (length $-l_{g}$, interior diameter $-d_{b}$, initial chamber length $-l_{0}$ ), projectile characteristics (total length $-l_{b}$, length of the cone part $-l_{h}$, base diameter $-d_{b}$, mass $-q$ ) and the charge characteristics (propellant impulse $-\lambda$, burning rate $-\beta$, diameter of charge grain $-d_{c}$, grain geometrical coefficient $-\theta$ ) are chosen then the target of interior ballistic calculations is to determine the propellant amount $(\omega)$ needed to accelerate the bullet to the desired muzzle velocity with an appropriate estimation of the environment reaction to the projectile motion in the gun tube. So a good model for the water resistance force is very important factor. Thus numerical simulations based on a good enough governing Eqs. (1) of the problem will give the dependence of the muzzle velocity on the charge mass. This is also the primary interest of the gun designing.

First of all let consider the acting force on the bullet created by the propellant combustion in the gun chamber. This force obviously equals the pressure on the bullet base times its area. So we have to obtain the pressure distribution in the barrel length. Here we need some assumptions. The first is that the gas (the product of the propellant combustion at any time $t$ ) is homogeneous in the space behind the bullet, so

$$
\rho=\rho(z, t),
$$

where $z$ is the location of the center of the gas mass. This assumption is reasonable because the combustion process of the charge lasts in an interval measured by milliseconds and it occurs in a very small volume. Denoting $\eta$ as the length of the part of the charge that had been burned at time $t\left(\eta=0\right.$ at $t=0$ and $\eta=-l_{0}$ at the burnout) one has

$$
z=(x-\eta) / 2 \text {. }
$$


Using this relationship after some transformations we can get

$$
\frac{V_{z}}{z}=\frac{V}{x}
$$

where $V_{z}$ is the velocity of the gas center of mass. For gas motion in the volume behind the bullet in the barrel tube we apply the one-dimensional Euler equation neglecting the gravity force of the gas

$$
\frac{d p}{d z}=-\rho \frac{d V}{d t}=-\rho\left(\frac{\partial V_{z}}{\partial t}+V_{z} \frac{\partial V_{z}}{\partial z}\right)
$$

Substituting $V_{z}$ from (4) into (5) and after some transformations we can get

$$
\frac{d p}{d z}=-\rho \frac{z}{x} \ddot{x}
$$

It is obvious that the exact expression for the gas density at any time is

$$
\rho=\omega|\eta| /[S(x-\eta)]=\omega|\eta| /[2 z S] .
$$

where $\omega$ is the charge mass before the ignition. However here we take

$$
\rho=\omega /\left[S\left(x+l_{0}\right)\right]
$$

that equals to the gas density at the moment of the charge burnout. This approximation is quite reasonable when the charge combustion time is very small. So Eq. (6) now can be rewritten in the form

$$
\frac{d p}{d z}=-\frac{\omega}{S\left(x+l_{0}\right)} \frac{z}{x} \ddot{x}
$$

Integrating (7) in respect with $z$ and doing some transformations we can get

$$
p(z, t)=p_{S}+\frac{\omega \ddot{x}}{2 S\left(x+l_{0}\right)}\left(x-\frac{z^{2}}{x}\right),
$$

From $(8)$, it is easy to obtain the pressure at the breech $\left(p_{B}\right)$ and the average pressure behind the bullet $(\bar{p})$ in the form

$$
p_{B}=p_{S}+\frac{\omega \ddot{x}}{2 S\left(x+l_{0}\right)} x, \bar{p}=\frac{1}{x+l_{0}} \int_{0}^{x+l_{0}} p(z) d z=p_{S}+\frac{\omega \ddot{x}}{2 S\left(x+l_{0}\right)}\left[x-\frac{\left(x+l_{0}\right)^{2}}{3 x}\right] .
$$

It is shown from (8) and (9) that at all time $p_{B}>\bar{p}>p_{S}$. Note that expressions (8) and (9) are more exact than that given in [2]. Now we use (1) to determine $p_{S}$. We have

$$
\ddot{x}=\left(p_{S} S-R\right) /\left(q+m_{a d}\right) \text {. }
$$

Substitute this expression into (9) we get $\bar{p}=p_{S}+\frac{\omega}{2 S\left(x+l_{0}\right)}\left(x-\frac{\left(x+l_{0}\right)^{2}}{x}\right) \frac{p_{S} S-R}{q+m_{a d}}$. Hence

$$
p_{S}=(\bar{p}+R \Delta) /(1+S \Delta)
$$


where $\Delta=\frac{\omega}{2 S\left(x+l_{0}\right)\left(q+m_{a d}\right)}\left(x-\frac{\left(x+l_{0}\right)^{2}}{3 x}\right)$. To complete the system of governing equations of the problem we add some results of the theory of combustion [5]. They are as follows

$$
d_{c} \dot{f}=-\beta p_{B}, \quad \bar{p}=\frac{\omega \lambda(1-f)(1+\theta f)}{S\left(x+l_{0}\right)} .
$$

The algorithm for calculating the solution of the interior ballistic problem now can be presented by the following cycle

$$
f \rightarrow \bar{p} \rightarrow p_{S} \rightarrow \ddot{x} \rightarrow p_{B} \rightarrow f \ldots
$$

To resume the above discussions we write out the system of equations that should be integrated in implementation of the above cycle

$$
\begin{aligned}
& d_{c} \dot{f}=-\beta p_{B}, \\
& \bar{p}=\frac{\omega \lambda(1-f)(1+\theta f)}{S\left(x+l_{0}\right)}, \\
& p_{S}=(\bar{p}+R \Delta) /(1+S \Delta), \\
& \ddot{x}=\left(p_{S} S-R\right) /\left(q+m_{a d}\right), \\
& p_{B}=p_{S}+\frac{\omega \ddot{x}}{2 S\left(x+l_{0}\right)} x .
\end{aligned}
$$

The initial conditions at $t=0$ for this system are

$$
x=0, \quad V=0, \quad f=1 .
$$

It should be noted that Eqs. (11), (12), (13) and (15) are valid only for $f>0$. After the moment of charge burnout $(f=0)$, the above system equations should be replaced by the following one

$$
\begin{gathered}
\ddot{x}=\left(p_{S} S-R\right) /\left(q+m_{a d}\right), \\
p_{S}=\bar{p}_{*}\left(\rho / \rho_{*}\right)^{\gamma},
\end{gathered}
$$

where $p_{*}$ and $\rho_{*}$ are the average pressure and gas density respectively in the barrel at the burnout moment of the charge whilst $\gamma$ is the adiabatic compressible coefficient of the gas. Relationship (18) is valid with the assumption that the expansion of the gas behind the bullet in the barrel after the burnout is isentropic [6]. This assumption can be adopted because the expansion process happens in a very short time interval and in a very small volume so the heat transfer from the gas to the environment may be ignored.

Next consider the resistance force $R$ exerted on the bullet by the water. This force is much greater than that similar when shooting in the air because both the water density and viscosity are very significant in comparison with those of the air. The resistance force in this case can be split into two main components $R=R_{1}+R_{2}$. The first component is related to the water hydrostatic pressure increasing with the depth and the second term is the essential friction caused by the bullet motion and water viscosity. We estimate the 
first component by

$$
R_{1}=\int_{S_{h}}-P_{h} \sin \alpha d s,
$$

where $P_{h}$ is the hydrostatic pressure of water at depth $h, \alpha$ is the cone angle of the bullet head and $S_{h}$ is its area. It is easy to get

$$
R_{1}=-P_{h} S .
$$

Before estimation of the second term we make some notes as follows. In fact during the motion of the bullet in the barrel, there is an amount of the gas moves out through the very small gap between the bullet and the interior surface of the barrel. This motion makes the water flow fully turbulent right it begins. So when estimate the friction force of the water we should take this into account. In our case the water flow with very good approximation can be regarded as asymmetric. So for calculating the friction force exerted by water on the bullet we can rely on the theory of boundary layer that presented in detail in [7]. For our problem the model of turbulent flow over a flat plate is applicable. So if the friction force acted on the bullet is presented in the form

$$
R_{2}=C_{f} S \rho V^{2} / 2,
$$

where $C_{f}, \rho, S$ and $V$ are the friction coefficient, water density, area of the bullet base and bullet velocity respectively then for $C_{f}$ we can take the following empirical formulae

$$
C_{f}=\frac{0.455}{[\log (R e)]^{2.58}}, \quad 5.10^{5}<\operatorname{Re}<10^{9},
$$

where the Reynolds number is defined by $R e=\rho l_{b} * V / \mu$ with $\mu$ being the water dynamical viscosity. In our case the Reynolds number reaches value of $1.2 \times 10^{6}$ at the muzzle velocity around $240 \mathrm{~m} / \mathrm{s}$.

To complete our model we discuss the added mass $m_{a d}$ in Eqs. (14) and (17). When the bullet moves in the barrel tube it always expels some water amount from the barrel. We consider this amount as the added mass to the bullet mass at any time moment. It is obvious that this mass exists when $x+l_{b}<l_{g}$ or $x+l_{b}-l_{h}<l_{g}$. And we have

$$
m_{a d}=\left\{\begin{array}{lr}
\rho S\left(l_{g}-l_{b}-x+2 l_{h} / 3\right) & \text { if } x+l_{b}<l_{g} \\
\rho\left[S\left(l_{g}-x-l_{d}\right)-\pi \tan ^{2} \alpha\left(l_{h}^{3}-\left(l_{g}-x-l_{d}\right)^{3}\right) / 3\right] & \text { if } x+l_{b}-l_{h}<l_{g}
\end{array}\right.
$$

Thus our problem of the interior ballistics consists of (11) - (16) for the stage before the charge combustion process ends, and of (17), (18) after the burnout. This system of equations can be integrated by any technique available for ordinary differential equations provided in [6], for example by Runge-Kutta fourth order procedure.

\section{RESULT OF SIMULATION AND DISCUSSION}

The simulation was carried out for two bullet models with different total length $\left(l_{b}\right)$ and weight $(q)$. For each parameter of the charge such as the mass $(\omega)$, grain diameter $\left(d_{c}\right)$, and grain geometrical coefficient $(\theta)$ two values were chosen for simulations. The typical variation of the gas pressure at the bullet base $p_{S}$ (in pascals) as a function of its location $x$ (in meters) in the barrel is shown in Fig. 2. The breech pressure as well as the 
average one has the similar behavior too. It is not necessary that the maximum pressure occurs at the burnout. In fact this peak is usually reached before the solid grains of the charge completely have been transformed into gas. It is because those pressures at any time depend not only on the part of the charge that has been transformed into gas but also on the volume behind the bullet in the barrel. The monotonous variation of the bullet velocity in the barrel tube is presented in Fig. 3. The behavior of the pressure and velocity given in Fig. 2 and Fig. 3 perfectly reflects their features in fact.

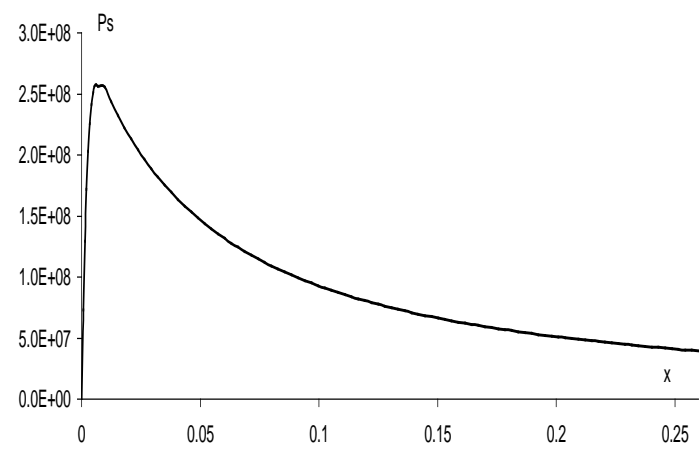

Fig. 2. Variation of $p_{s}(x), l_{b}=0.115$, $\omega=0.0005, d_{c}=0.0005, \theta=1.0$

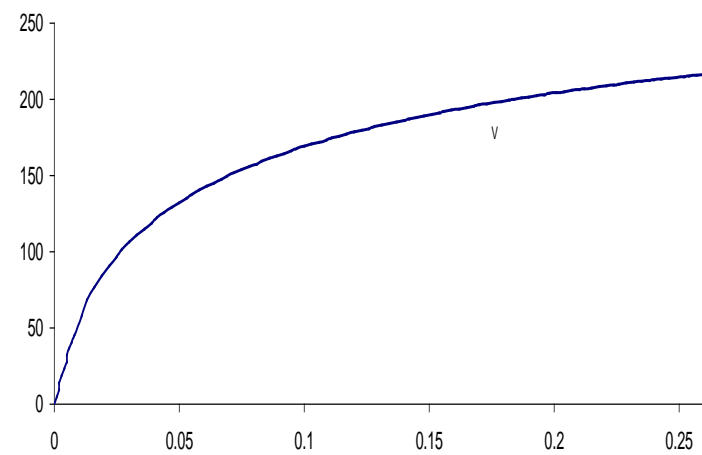

Fig. 3. Variation of $V(x), l_{b}=0.115$, $\omega=0.0005, d_{c}=0.0005, \theta=1.0$

It is interesting to elucidate the effect of water environment on interior ballistic characteristics of the gun. For this purpose the above system of equations is integrated with and without the resistance force $R$. The difference between these cases is shown in Figs. 4 and 5.

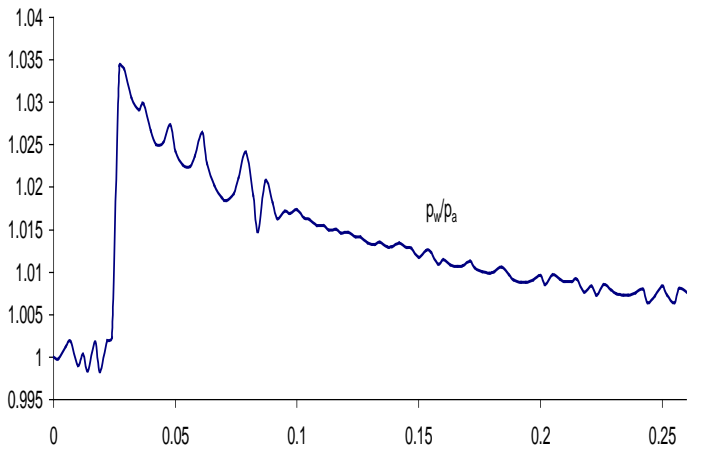

Fig. 4. Ratio of pressures $p_{w}(x) / p_{a}(x), l_{b}=$ $0.14, q=0.026, \omega=0.0005, d_{c}=0.001, \theta=1.0$

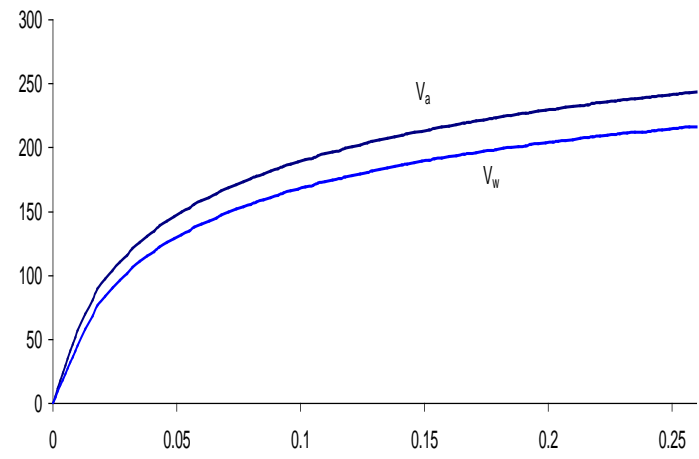

Fig. 5. Velocity $V_{a}(x)$ and $V_{w}(x), l_{b}=$ $0.115, \omega=0.0005, d_{c}=0.0005, \theta=0.0$

As indicated in Fig. 4, for all the simulations, at all the locations the water resistance makes the pressure always slightly higher than that of the nonresistance case. This is in the accordance with (10) as well as experiment data. Meantime the water resistance decreases the bullet velocity at all the sections as shown in Fig. 5. 
In Tab. 1 the comparison of the calculated muzzle velocity $(\mathrm{m} / \mathrm{s})$ with experimental data is provided for two cases shooting in air and in water.

Table 1. $V\left(l_{g}\right)$ for $l_{g}=0.26, l_{b}=0.115, q=0.0204, \omega=0.0005, d_{c}=0.0005, \theta=0.0$

\begin{tabular}{|c|c|c|c|}
\hline \multicolumn{2}{|c|}{ Calculated } & \multicolumn{2}{c|}{ Experiment } \\
\hline In air $(\mathrm{A})$ & In water $(\mathrm{W})$ & $(\mathrm{A})$ & $(\mathrm{W})$ \\
\hline 243.6 & 231.2 & 241 & 236.5 \\
\hline
\end{tabular}

The error for case $\mathrm{A}$ is about $1.1 \%$ whilst for case $(\mathrm{W})$ it equals approximately $2.2 \%$.

It is worth noting that in practice to calculate the solution of a ballistic problem, especially exterior one, a suitable law for the resistance force for a concrete projectile is chosen. Then for fitting the calculated results with experimental data the so-called drag coefficient is used to express the drag of the projectile by multiplying this coefficient and the chosen law together. In our case, if we represent

$$
R_{2}=\bar{c} C_{f} S \rho V^{2} / 2,
$$

where $\bar{c}$ is the above mentioned drag coefficient for the bullet then $\bar{c}$ can be chosen to make the calculated muzzle velocity of the bullet equals to its measured value. Doing so, for the bullet and the charge of the following characteristics (denoted by B1)

$$
l_{b}=0.115, l_{h}=0.025, d_{b}=0.0057, q=0.0204,
$$

we get $\bar{c}=0.68245$. Analogously we have $\bar{c}=0.37145$ for the bullet B2 with its parameter set: $l_{b}=0.14, l_{h}=0.025, d_{b}=0.0057, q=0.026$. Using these coefficients we can simulate the motion of bullets in the barrel. The Tab. 2 represents the simulation results for bullets $\mathrm{B} 1$ and $\mathrm{B} 2$ with different values of $d_{c}$ and $\theta$

Table 2. The muzzle velocity of underwater shooting

\begin{tabular}{|c|c|c|c|c|}
\hline Bullet & $d_{c}=0.001, \theta=0$ & $d_{c}=0.001, \theta=1$ & $d_{c}=0.0005, \theta=0$ & $d_{c}=0.0005, \theta=1$ \\
\hline B1 & 228 & 238.5 & 231 & 233.5 \\
\hline B2 & 206.5 & 214.5 & 210.5 & 212 \\
\hline
\end{tabular}

In Tab. 3 shown the impact of the extra weight and length of B2-bullet on its decrease of velocity in comparison with B1-bullet.

Table 3. The decrease of the muzzle velocity related to the extra weight

\begin{tabular}{|c|c|c|c|c|}
\hline Muzzle deflection & $\begin{array}{c}d_{c}=0.001, \\
\theta=0\end{array}$ & $\begin{array}{c}d_{c}=0.001, \\
\theta=1\end{array}$ & $\begin{array}{c}d_{c}=0.0005, \\
\theta=0\end{array}$ & $\begin{array}{c}d_{c}=0.0005, \\
\theta=1\end{array}$ \\
\hline$\delta V(\%)$ & 9.43 & 10.06 & 8.87 & 9.21 \\
\hline$\delta V / \mathrm{grm},(\delta V / \mathrm{cm})$ & $1.68(3.77)$ & $1.80(4.03)$ & $1.58(3.55)$ & $1.64(3.68)$ \\
\hline
\end{tabular}




\section{CONCLUSION}

The interior ballistic problem for underwater shooting is considered in details in this paper. To work out the dependence of the pressure on the base of the projectile on the combustion of the charge as well as the resistance of the water environment on the projectile motion in the barrel tube are crucial for solving the problem. A software suitable for gun-bullet design is created. The results of simulation based on this software are quiet good in comparison with experiment data for the considered gun.

\section{ACKNOLEDGEMENT}

The authors acknowledge the support by the VAST.HDN.01/15-16 project

\section{REFERENCES}

[1] R. I. McCoy. Modern exterior ballistics. Schifter Military History, Atglen, PA, (1999).

[2] D. E. Carlucci and S. S. Jacobson. Ballistics: theory and design of guns and ammunition. Taylor \& Francis Group, LLC, (2007).

[3] F. Rebbins. Interior ballistics course notes. Self published, Aberdeen, MD, (2002).

[4] J. Corner. Theory of the interior ballistics of guns. John Wiley and Sons, NY, (1950).

[5] N. Kubota. Propellant and explosives. Wiley VCH, New York, (2002).

[6] T. V. Tran. Fundamentals of gas dynamics. VNUH Publishing House, (2004). (in Vietnamese).

[7] H. Schlichting. Boundary-layer theory. McGraw-Hill, 6-th edition, (1968). 